\title{
Chemoprevention of Barley and Sage against acrylamide-Induced genotoxic, biochemical and histopathological alterations in rats
}

\author{
Sekena, H. Abd El-Aziem', Mosaad A. Abdel-Wahab², Mahmoud A. M² Azza M. \\ Hassan ${ }^{4}$ and Karima, F. Mahrous ${ }^{1}$
}

\author{
${ }^{1}$ Department of Cell Biololgy, ${ }^{2}$ Department of Food Toxicology \& Contaminants National \\ Research Centre, Dokki, Cairo, Egypt. and ${ }^{3}$ Department of Pathology. Faculty of Vet. Med. \\ Cairo Univ. Egypt and ${ }^{4}$ Forensic Medicine \& Toxicology Department, Faculty of Medicine for \\ girls, Al Azhar University, Cairo, Egypt.
}

\begin{abstract}
Acrylamide (ACR) has recently been found in fried and backed foods, suggesting widespread public exposure. ACR is an industrial chemical material that causes neurotoxicity in humans and was designated as a probable human carcinogen by IARC and USEPA. The aim of the present study was to evaluate the protective effects of barley and sage against ACR mutagenicity and biochemical and histopathological changes in rats. Forty mature male rats were divided into eight groups and were fed barley and/or sage-supplemented diet (5\%) with or without ACR $(50 \mathrm{mg} / \mathrm{kg}$ b.w). The biochemical results revealed that ACR increased Alanine amino transferase (ALT), Aspartate amino transferase (AST), triglycerides (TG), cholesterol and uric acid. Micronucleated polychromatic erythrocytes and chromosomal aberrations in somatic and germ cells were significantly increased in ACR-treated animals. Severe pathological lesions included testicular degeneration, oedema, spermatid giant cells formation and necrosis of spermatid cells were found in the testis of ACR-treated group. The kidney of this group showed degenerative changes. Cotreatment with barley and/or Sage and ACR resulted in a significant improvement in all the parameters tested. It could be concluded that these plants contain antioxidant compounds and may be useful when add as food additive to the food cooked on a higher temperature.
\end{abstract}

Key words: Acrylamide, Barley, Sage, chromosomal aberrations, kidney, testis.

\section{Introduction}

In the year 2002 the Swedish National Food Authority reported the presence of elevated levels of acrylamide (ACR) in certain types of food processed at high temperature. Since then ACR has been found in a lot of cooked and heat-processed foods in other countries, including Netherlands, Norway, Switzerland, United States, United Kingdom (Shelby, 2004), and Egypt. Human exposed to ACR through the consumption of heat-cooked food such as potato crisps, chips, cocktail snacks and gingerbread. Chronic ACR exposure has been associated with increased incidence of mesothelioma, cancer of central nervous system, thyroid gland and other endocrine glands, mammary glands and reproductive tracts in rats (Johnson et al., 1986). ACR is regarded as a potential mutagen based on experimental evidence that it can bind to DNA (Sega, 1990). ACR undergoes biotransformation by conjugation with glutathione (Miller, 1982) or reduction by microsomal cytochrome P-450 (Anzenbacher and Anzenbacherova, 2001). The major metabolite of ACR, glycidamide is an epoxide that may be more critical for carcinogenic and genotoxic properties in animals than the parent compound (Segerback et al., 1995).

ACR exposure in hamsters caused testicular atrophy with damage to spermatids and mature spermatozoa (Sakamoto et al., 1988). Reduced sperm motility and impaired fertility have also been reported in treated mice and rats 
(Zenick et al., 1986). ACR caused chromosomal aberrations in mammalian cells in vitro and in vivo (Backer et al., 1989, Collins et al., 1992). Micronuclei were found in mouse bone-marrow cells and splenocytes following exposure to ACR (Collins et al., 1992). ACR induces pathological lesions in different body organs including variety of tumors in rats and mice (U.S.EPA, 1994; ACGIH, 1991). In human, inadequate evidence is available from human studies regarding the tumor formation by acrylamide.

Previous studies indicated that the leaves of sage (Salvia officinalis L.) are well known for their anti-oxidative properties (Chipault et al., 1956; Baricevic et al., 1996), used in the food processing industry but applicable also to the area of human health (Pearson et al., 1997). The plant is reported to have a wide range of biological activities, such as anti-bacterial, fungistatic, virustatic, astringent, eupeptic and anti-hydrotic effects (Dobrynin et al., 1976, Cherevaty et al., 1980). Experimental studies on sage extracts or sage essential oil showed hypotensive properties, central nervous system-depressant actions and antispasmodic activity (Newall et al., 1996). Moreover, the antimutagenic potential of sage extracts was demonstrated on Escherichia coli repair proficient strains (Baricevic et al.,1996; Filipic and Baricevic 1997 and1998).

Barley (Hordeum vulgare L.) grains are relatively rich in dietary fibers such as glucan, arabinoxylans and cellulose. The consumption of glucan-rich diet results in several beneficial physiologic effects due to a relatively high concentration, soluble state and high molecular weight of this polysacride (Dongowski, et al., 2002). The extractability and viscosity of glucan are influenced by both the technologic conditions in the gastrointestinal tract (Robertson, et al., 1997, Johansen, et al., 1993). Generally, the previous studies concluded that dietary fiber-rich barleycontaining diets have beneficial physiologic effects. The aim of the present study was to evaluate the protective effects of barley and sage against ACR-induced toxicity, mutagenicity biochemical and pathological changes in rats.

\section{Materials and methods Acrylamide}

Acrylamide was purchased from MERCK-Schuchardt Chemical Co. (Hohenbrunn, Germany) with molecular formula $\mathrm{NH} 2-\mathrm{C}=\mathrm{O}-\mathrm{CH}-\mathrm{CH} 2$, with purity $>99 \%$.

Kits: Transamianse (ALT and AST) were purchased from Randex Laboratories (San Francisco, CA, USA). Cholesterol, triglycerides and uric acid were obtained from Biomerieux, Laboratory of Reagents and Products (Marcy Létoile, France).

\section{Plants}

Barley was purchased from local market at Cairo, Egypt. Whereas, Sage (Salvia officinalis) was supplied by the Department of crop production, Faculty of Agriculture, Ain-Shams University.

\section{Animals and Treatments}

Experimental Animals: Ten-week-old, adult male Sprague-Dawley rats weighting 180$200 \mathrm{~g}$ (purchased from Animal House Colony, Giza, Egypt) were maintained on standard lab diet (Protein: 16.04\%; Fat: 3.63\%; Fiber: $4.1 \%$, and metabolic energy: $0.012 \mathrm{MJ})$ and water ad libitum at the Animal House Lab., National Research Center, Dokki, Cairo, Egypt. After an acclimation period of 1 week, animals were distributed into eight groups (5 rats /group) and housed in stainless steel cages housed in a temperature-controlled $\left(23 \pm 1{ }^{\circ} \mathrm{C}\right)$ and artificially illuminated (12 hr dark/light cycle) room free from any source of chemical contamination.

Experimental Design: After an acclimation period of one week, animals were divided into eight treatment groups (5 rats /group). Groups 1-4 were fed on barley diet only for three months whereas, groups 5-8 were fed on the standard lab diet for the same period. At the end of the 3rd month, animals within all groups were treated for five days as follow: group (1) control, group (2) treated orally with ACR (50 mg/kg b.w), group (3) fed on ad lib barley diet, group (4) fed on barley diet and treated orally with ACR, group (5) fed on standard lab diet 
supplemented with 5\% dried sage leaves, group (6) fed on lab diet supplemented with dried sage leaves and treated orally with ACR, group (7) fed on barley diet supplemented with 5\% dried sage leaves, and group (8) fed on barley diet supplemented with sage leaves and treated orally with ACR.

At the end of the experiment period, blood samples were collected from the retro-orbital venous plexus under diethyl ether anesthesia. Blood samples were left to clot and the sera were separated using cooling centrifugation and stored at $-20 \mathrm{OC}$ until analysis. The activities of Alanine amino transferase (ALT) and Aspartate amino transferase (AST) were determined according to the method recommended by The Committee on Enzymes of the Scandinavian Society for Clinical Chemistry and Clinical Physiology (1974). Cholesterol was determined in serum according to Charles and Richmond (1974), triglycerides (TG) was determined in serum according to Wahlefeld, (1974) and uric acid was determined in serum according to Haisman and Muller (1977). Then animals were injected intraperitonealy (i.p) with 0.1 $\mathrm{ml}$ of $0.5 \%$ of colchicine $3 \mathrm{~h}$ before sacrifice. The femora and testis were removed from all animals and prepared for the micronucleus and chromosomes studies as follow:

For micronucleus analysis: Slides were prepared according to Salamone et al. (1980) The bone marrow cells were mixed with one drop of fetal calf serum and smeared on clean glass slides. The slides were air dried and fixed in methyl alcohol for 5 min. then stained with 5\% Giemsa and mounted with DPX. Two thousands PCEs/animal were recorded.

For chromosomal analysis: The bone marrow was prepared, stained and scored according to Preston et al. (1987). To study the effects of different treatments on the mitotic activity of the bone marrow, 100 cells per animal were counted and the number of dividing cells including prophases and metaphases were scored. The testis of the same animals were removed and slides were prepared according to the method adopted by Russo (2000). Seventy- five metaphase spreads were examined in spermatocytes.

\section{Histopathological examination: -}

Samples of testis and kidneys were collected from each animal within each treatment immediately after scarification and fixed in $10 \%$ neutral buffered formalin. Tissue specimens were processed routinely for paraffin sections of 4-5 micron thickness, stained with Hematoxylin and Eosin (H\&E) according to Bancroft et al. (1996). Whereas, Crystal violet-eosin stain was used to determine the normal and abnormal spermatozoa in the testis (Deeb, 1976).

\section{Statistical analysis:}

All data for biochemical analysis were statistically analyzed using the General Linear Model Procedure of the Statistical Analysis System (SAS, 1982). The significance of the differences among treatment groups was determined by Waller-Duncan k-ratio (Waller and Duncan, 1969). All statements of significance were based on probability of $\mathrm{P}<0.05$. For micronucleus test and chromosomal aberrations study, the comparison of reliability analysis (F. test) was applied (Snedecor and Cochran, 1961).

\section{Results}

Results of the biochemical analysis (Table 1) revealed that treatment with ACR caused a significant increase in all tested parameters. Sage alone resulted in a significant decrease in all tested parameters except AST which was comparable to the controls. Rats fed barley diet only showed a significant decrease in TG, Cholesterol and uric acid levels in serum, whereas, ALT and AST were comparable to the control. Both sage and barley were comparable to the control regarding AST, whereas the other biochemical parameters were found to decrease than the control values. Addition of sage to the diet of animals treated with ACR resulted in a significant improvement in all the parameters tested. This treatment was effective in decreasing the elevated levels of all the biochemical parameters although they were still differ significantly than the control. Animals fed barley diet and received ACR were comparable to the 


\section{Sekena, H. Abd El-Aziem et al}

control regarding ALT while the other parameters were still significantly higher than the control. Animals treated with ACR and received barley plus sage showed a normal AST, TG and cholesterol levels whereas, a significant decrease and a significant increase was found for ALT and uric acid respectively.

\section{Micronucleus study}

Data presented in table (2) and fig. (1a) show the effects of different treatments on polychromatic erythrocytes (PCEs) in bone marrow of rats. Treatment with ACR resulted in a significant increase $(\mathrm{P}<0.01)$ in the micronucleated polychromatic erythrocyte (MnPCEs) whereas; no significant differences were noticed in the MnPCEs between the control group and groups treated with barley or sage. Animals fed barley for three months showed an inhibition of MnPCEs reached $69.3 \%$. Meanwhile, sage alone showed a significant $(\mathrm{P}<0.01)$ inhibition in MN-PCEs $(35.42 \%)$.

\section{Somatic cells: -}

In the present study, the chromosomal examination showed that structural aberrantions include chromatid and chromosomal gaps (Fig. 1c), deletions and end to end associations (Fig. 1d), chromatid and chromosomal breaks (Fig. 1e), and fragments (Fig. 1f). Numerical aberrations were studied represented by polyploidy (Table 3) (Fig. 1g).

Statistical analysis showed a significant increase $(\mathrm{P}<0.001)$ in total structural chromosomal aberrations with or without gaps and total aberrations in ACRtreated group compared with the control group. The total structural aberrations and total aberrations recorded a significant increase $(\mathrm{P}<0.01)$ in $\mathrm{ACR}$-sage treated group compared with sage group. These differences in total structural aberrations were also significant $(\mathrm{P}<0.05)$ compared with those of animals fed barley alone or barley plus sage (Table 3 ).

The main frequent types of structural aberrations in the animals treated with ACR plus barley was chromosomal breaks which found to be significant $(\mathrm{P}<0.05)$ whereas, the other types of aberrations were nonsignificant. Animals treated with ACR plus sage showed a significant increase in case of gaps and the other types of aberrations ( $p<0.01$ and 0.05 respectively). Animals pretreated with barely or fed barley supplemented with 5\% sage, or those fed control diet supplemented by 5\% sage showed an inhibition in chromosomal aberrations in bone marrow $(59.6 \%, 63.1 \%$, and $37 \%$ respectively. Animals fed barley supplemented with 5\% sage showed insignificant changes in all other type of aberrations.

Spermatocytes: -

Concerning the spermatocytes abnormalities, data presented in table (4) and fig (2) clearly indicated that treatment with ACR resulted in a significant increase $(\mathrm{P}<0.01)$ in total structural aberrations. The main types of abnormalities observed were chain (fig. 2b), autosomal univalent (fig. 2c), X-Y univalent and polyploidy (fig. 2d). Animals treated with sage, barley or barley plus sage showed nonsignificant increase compared with the control group. Treatment with barley, sage or barley plus sage succeeded to decrease significantly the abnormalities of spermatocytes (71.4, 46 and $85.7 \%$ respectively) although some abnormalities such as $\mathrm{x}-\mathrm{y}$ univalents, chain, autosomal univalents and total structural aberrations were still significant in the animals received the combined treatment $(\mathrm{P}<0.05)$.

Results in table (4) indicated the numerical aberrations recorded. These results showed a significant increase in ACR group compared with the control $(\mathrm{P}<$ $0.01)$. Meanwhile, the pretreatment with barley or sage plus barley inhibited the total numerical aberrations.

\section{Histopathological results:-}

The histopathological examination of the animal treated with ACR alone revealed severe pathological lesions in the testicular tissues. A large number of residual bodies in the lumen of the seminiferous tubules were noticed (Fig. 3a). Some of the seminiferous tubules were completely devoid of mature sperms while others showed few number as demonstrated by positive reaction of the mature sperms to crystal violet stain (Fig.3b). Necrotic spermatocytes with chromatolysis were also noticed (Fig3, c,d). The most common 
testicular lesion in the animals in this group was the presence of varied number of spermatid giant cells. Some of the seminiferous tubules appeared with single giant cell with foamy cytoplasm (Fig. 4a) while the others were impacted with 3-6 spermatid giant cells (Figs. 4b,c). The nuclei of these cells were varied in number and ranged from 2-5 nuclei (Figs. 4d,e). Interstitial oedema was also observed in the testis with spermatid giant cells (Fig. 4f).

The animal fed barley and treated with ACR showed noticeable decrease of the testicular lesions. The seminiferous tubules appeared impacted with mature spermatocytes, which confirmed by positive reaction to crystal violet (Fig. 5a,b). On the other hand, animals fed sage supplemented diet and treated with ACR appeared with low number of mature spermatocytes but without obvious pathological lesions (Fig. $5 \mathrm{c}, \mathrm{d})$.

The kidneys of the animals in ACRtreated group showed mild degenerative changes with shrunken glomerular capillary tufts (Fig. 6a). Animals fed barley and/or sage showed no obvious pathological lesions in the testicular and kidney tissues. The kidneys of both groups and received ACR were apparently normal (Fig 6b).

Table (1). Effect of ACR with or without barley or sage on serum biochemical parameters in rats

\begin{tabular}{|c|c|c|c|c|c|c|c|c|}
\hline & Control & ACR & Barley & $\begin{array}{l}\text { ACR + } \\
\text { Barley }\end{array}$ & Sage & $\begin{array}{l}\mathrm{ACR}+ \\
\text { Sage }\end{array}$ & $\begin{array}{c}\text { Barley + } \\
\text { Sage }\end{array}$ & $\begin{array}{c}\text { ACR + } \\
\text { Barley + } \\
\text { Sage }\end{array}$ \\
\hline $\begin{array}{l}\text { ALT } \\
(\mathrm{U} / \mathrm{L})\end{array}$ & $\begin{array}{l}36.52 \mathrm{a} \\
\pm 0.85\end{array}$ & $\begin{array}{l}46.99 \mathrm{c} \\
\pm 1.53 \\
\end{array}$ & $\begin{array}{l}36.84 \mathrm{a} \\
\pm 0.68\end{array}$ & $\begin{array}{l}36.33 \mathrm{a} \\
\pm 0.61\end{array}$ & $\begin{array}{l}33.65 \mathrm{~b} \\
\pm 0.68\end{array}$ & $\begin{array}{l}34.03 b \\
\pm 0.75\end{array}$ & $\begin{array}{l}34.28 b \\
\pm 1.29\end{array}$ & $\begin{array}{l}31.24 \mathrm{~d} \\
\pm 0.95\end{array}$ \\
\hline $\begin{array}{l}\text { AST } \\
(\mathrm{U} / \mathrm{L})\end{array}$ & $\begin{array}{l}42.17 \mathrm{a} \\
\pm 0.79\end{array}$ & $\begin{array}{l}56.50 \mathrm{c} \\
\pm 1.18 \\
\end{array}$ & $\begin{array}{l}43.33 b \\
\pm 0.88\end{array}$ & $\begin{array}{l}44.67 \mathrm{~b} \\
\pm 1.33\end{array}$ & $\begin{array}{l}41.08 \mathrm{a} \\
\pm 1.02\end{array}$ & $\begin{array}{l}45.33 b \\
\pm 1.05\end{array}$ & $\begin{array}{l}41.62 \mathrm{a} \\
\pm 0.75\end{array}$ & $\begin{array}{l}41.57 \mathrm{a} \\
\pm 0.79\end{array}$ \\
\hline $\begin{array}{c}\mathrm{TG} \\
(\mathrm{mg} / \mathrm{dl})\end{array}$ & $\begin{array}{l}55.00 \mathrm{a} \\
\pm 1.41\end{array}$ & $\begin{array}{l}71.50 \mathrm{c} \\
\pm 1.41\end{array}$ & $\begin{array}{l}43.17 \mathrm{~b} \\
\pm 1.08\end{array}$ & $\begin{array}{c}58.67 \mathrm{ad} \\
\pm 1.05\end{array}$ & $\begin{array}{l}42.83 \mathrm{~b} \\
\pm 1.35\end{array}$ & $\begin{array}{l}62.17 \mathrm{~d} \\
\pm 1.99\end{array}$ & $\begin{array}{l}39.67 \mathrm{~b} \\
\pm 0.67\end{array}$ & $\begin{array}{l}56.17 \mathrm{a} \\
\pm 1.30\end{array}$ \\
\hline $\begin{array}{c}\text { Choleste- } \\
\text { rol } \\
(\mathrm{mg} / \mathrm{l}) \\
\end{array}$ & $\begin{array}{l}68.00 \mathrm{a} \\
\pm 1.41\end{array}$ & $\begin{array}{l}91.67 \mathrm{c} \\
\pm 1.82 \\
\end{array}$ & $\begin{array}{l}56.00 \mathrm{~b} \\
\pm 1.15\end{array}$ & $\begin{array}{c}69.83 \mathrm{ad} \\
\pm 2.09\end{array}$ & $\begin{array}{l}57.50 \mathrm{~b} \\
\pm 1.43\end{array}$ & $\begin{array}{l}71.83 \mathrm{~d} \\
\pm 1.40\end{array}$ & $\begin{array}{l}54.17 \mathrm{~b} \\
\pm 1.17\end{array}$ & $\begin{array}{l}67.00 \mathrm{a} \\
\pm 0.97\end{array}$ \\
\hline $\begin{array}{l}\text { Uric Acid } \\
(\mathrm{mg} / \mathrm{dl})\end{array}$ & $\begin{array}{c}0.47 \mathrm{a} \\
\pm 0.02\end{array}$ & $\begin{array}{l}0.94 \mathrm{c} \\
\pm 0.02\end{array}$ & $\begin{array}{r}0.36 \mathrm{~b} \\
\pm 0.01\end{array}$ & $\begin{array}{l}0.64 d \\
\pm 0.02\end{array}$ & $\begin{array}{l}0.38 \mathrm{~b} \\
\pm 0.01\end{array}$ & $\begin{array}{r}0.66 \mathrm{~d} \\
\pm 0.01\end{array}$ & $\begin{array}{l}0.34 \mathrm{~b} \\
\pm 0.01\end{array}$ & $\begin{array}{c}0.61 \mathrm{~d} \\
\pm 0.02\end{array}$ \\
\hline
\end{tabular}

Within each raw, means superscript with the same letter are not significantly different $(\mathrm{P}<0.05)$

Table (2: The mean value of micronuclei detected in polychromatic erythrocytes of bone marrow cells induced ACR and the effect of antioxidants in rats

\begin{tabular}{|c|c|c|c|}
\hline groups & Mononuclear & Binuclei & Inhibition \% \\
\hline $\mathrm{M} \pm \mathrm{SE}$ & \multicolumn{2}{|c|}{$\mathrm{M} \pm \mathrm{SE}$} & \\
\hline control & $1.0 \pm 0.41$ & ------ & \\
\hline ACR (50mg/kg b.w) & $13.29 \pm 0.29^{* * *}$ & $1.0 \pm 0.41$ & \\
\hline barley & $1.75 \pm 0.85$ & ----- & $69.3 \%$ \\
\hline ACR+ barley & $4.5 \pm 0.86$ & $0.25 \pm 0.25$ & $35.42 \%$ \\
\hline Sage & $2.5 \pm 1.04$ & ---- & $86.5 \%$ \\
\hline ACR+ Sage & $9.25 \pm 1.11^{* *}$ & $0.5 \pm 0.29$ & \\
\hline Sage+ barley & $1.5 \pm 0.65$ & ----- & \\
\hline ACR+ Sage + barley & $3.25 \pm 0.25$ & ----- & \\
$=\mathrm{P}<0.05 * *=\mathrm{P}<0.01$
\end{tabular}


Table (3): Mean value of different chromosomal aberrations induced by ACR and the effect of antioxidants in bone marrow of male rats $(\mathrm{M} \pm \mathrm{SE})$

\begin{tabular}{|c|c|c|c|c|c|c|c|c|}
\hline \multirow{2}{*}{$\begin{array}{ll}\text { Types } & \text { of } \\
\text { aberrations } & \end{array}$} & \multicolumn{8}{|c|}{ Treatments } \\
\hline & control & ACR & barley & $\begin{array}{l}\text { ACR+ } \\
\text { barley }\end{array}$ & Sage & $\begin{array}{l}\text { ACR+ } \\
\text { Sage }\end{array}$ & $\begin{array}{l}\text { Sage+ } \\
\text { barley }\end{array}$ & $\begin{array}{l}\text { ACR+ } \\
\text { Sage+ } \\
\text { barley }\end{array}$ \\
\hline $\begin{array}{l}\text { Total } \\
\text { aberrations }\end{array}$ & $\begin{array}{ll}1.0 & \pm \\
0.41 & \end{array}$ & $\begin{array}{l}40.3 * \pm \\
1.8^{* * * *}\end{array}$ & $\begin{array}{ll}2.75 & \pm \\
0.47 & \end{array}$ & $\begin{array}{l}18.0 \text { * } \\
0.71^{*}\end{array}$ & $\begin{array}{l}4.0 \quad \pm \\
0.91\end{array}$ & $\begin{array}{l}27 \\
0.41^{* *}\end{array}$ & $\begin{array}{ll}3.25 & \pm \\
0.63 & \end{array}$ & $\begin{array}{ll}17.0 \quad \pm \\
4.4^{*}\end{array}$ \\
\hline $\begin{array}{l}\text { Total struct. } \\
\text { aberrations }\end{array}$ & $\begin{array}{l}0.75 \\
0.25\end{array}$ & $\begin{array}{l}32.75 \pm \\
1.31^{* * * *}\end{array}$ & $\begin{array}{ll}2.2 & \pm \\
0.48 & \end{array}$ & $\begin{array}{l}12.25 \pm \\
2.25^{*}\end{array}$ & $\begin{array}{l}3.75 \pm \\
0.25\end{array}$ & $\begin{array}{l}23.25 \pm \\
1.75^{* *}\end{array}$ & $\begin{array}{ll}2.5 & \pm \\
0.65 & \end{array}$ & $\begin{array}{l}13.75 \pm \\
2.1\end{array}$ \\
\hline $\begin{array}{l}\text { Total struct. } \\
\text { Ex. gap }\end{array}$ & $\begin{array}{ll}0.5 & \pm \\
0.29 & \\
\end{array}$ & $\begin{array}{l}25.25 \pm \\
1.31^{* * * *} \\
\end{array}$ & $\begin{array}{ll}1.5 & \pm \\
0.29 & \\
\end{array}$ & $\begin{array}{ll}10 & \pm \\
2.01^{*} & \\
\end{array}$ & $\begin{array}{l}2.25 \pm \\
0.85 \\
\end{array}$ & $\begin{array}{l}19 \pm \\
1.47^{* *}\end{array}$ & $\begin{array}{ll}1.5 & \pm \\
0.87 & \\
\end{array}$ & $\begin{array}{ll}10.5 & \pm \\
1.9^{*} & \\
\end{array}$ \\
\hline Gap & $\begin{array}{l}0.25 \\
0.25 \\
\end{array}$ & $\begin{array}{l}7.5 \quad \pm \\
0.64^{* * *}\end{array}$ & $\begin{array}{ll}0.75 & \pm \\
0.25 & \\
\end{array}$ & $\begin{array}{ll}2.5 & \pm \\
1.04 & \\
\end{array}$ & $\begin{array}{ll}1.0 \quad \pm \\
0.41\end{array}$ & $\begin{array}{ll}4.25 & \pm \\
63^{*} & \\
\end{array}$ & $\begin{array}{ll}1.0 \quad \pm \\
0.41 & \\
\end{array}$ & $\begin{array}{l}3.75 \\
1.25 \\
\end{array}$ \\
\hline Chromos. gaps & - & $\begin{array}{l}4.25 \\
0.75^{* *}\end{array}$ & - & $\begin{array}{ll}2.5 & \pm \\
0.96 & \end{array}$ & $\begin{array}{l}0.5 \pm \\
0.29\end{array}$ & $\begin{array}{l}2.75 \\
0.25^{*}\end{array}$ & $\begin{array}{ll}0.25 & \pm \\
0.25 & \end{array}$ & $\begin{array}{l}1.75 \\
0.63\end{array}$ \\
\hline Breaks & $\begin{array}{l}0.25 \\
0.25\end{array}$ & $\begin{array}{l}5.50 \pm \\
0.65^{* *}\end{array}$ & $\begin{array}{ll}0.5 & \pm \\
0.29 & \end{array}$ & $\begin{array}{ll}2.75 & \pm \\
0.48 & \end{array}$ & $\begin{array}{l}0.75 \pm \\
0.29\end{array}$ & $\begin{array}{l}3.75 * \\
0.93^{*}\end{array}$ & $\begin{array}{ll}0.75 & \pm \\
0.25 & \end{array}$ & $\begin{array}{l}2.25 \\
0.75\end{array}$ \\
\hline $\begin{array}{l}\text { Chromos. } \\
\text { breaks }\end{array}$ & $\longrightarrow$ & $\begin{array}{l}4.5 \quad \pm \\
0.29^{* *}\end{array}$ & $\begin{array}{ll}0.25 & \pm \\
0.25 & \end{array}$ & $\begin{array}{ll}2.25 & \pm \\
0.49^{*} & \end{array}$ & $\begin{array}{l}0.5 \pm \\
0.29\end{array}$ & $\begin{array}{ll}3.5 & \pm \\
0.64^{*} & \end{array}$ & $\begin{array}{ll}0.25 & \pm \\
0.25 & \end{array}$ & $\begin{array}{l}1.5 \\
0.65\end{array}$ \\
\hline Deletions & - & $\begin{array}{l}4.0 \quad \pm \\
0.41^{* *}\end{array}$ & - & $\begin{array}{l}1.5 \pm \\
0.65\end{array}$ & $\begin{array}{l}0.5 \pm \\
0.29\end{array}$ & $\begin{array}{ll}3.75 & \pm \\
0.85^{*} & \end{array}$ & $\begin{array}{ll}0.25 & \pm \\
0.25 & \end{array}$ & $\begin{array}{l}2.25 \\
0.85 \\
\end{array}$ \\
\hline Fragments & - & $\begin{array}{l}4.0 \quad \pm \\
0.41^{* *}\end{array}$ & 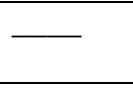 & $\begin{array}{ll}1.5 & \pm \\
0.65 & \\
\end{array}$ & $\begin{array}{l}0.5 \pm \\
0.29\end{array}$ & $\begin{array}{l}3.75 \\
0.85^{*}\end{array}$ & $\begin{array}{ll}0.25 & \pm \\
0.25 & \\
\end{array}$ & $\begin{array}{l}2.25 \\
0.85\end{array}$ \\
\hline E.E & $\begin{array}{l}0.25 \\
0.25 \\
\end{array}$ & $\begin{array}{l}3.25 \pm \\
0.48^{* * *}\end{array}$ & - & $\begin{array}{ll}1.0 & \pm \\
0.58 & \\
\end{array}$ & $\begin{array}{l}0.5 \pm \\
0.29\end{array}$ & $\begin{array}{ll}2.0 & \pm \\
0.91 & \\
\end{array}$ & $\begin{array}{ll}0.25 & \pm \\
0.25 & \\
\end{array}$ & $\begin{array}{l}0.75 \\
0.29 \\
\end{array}$ \\
\hline Polyploidy & $\begin{array}{l}0.25 \\
0.25 \\
\end{array}$ & $\begin{array}{l}7.5 \quad \pm \\
0.29 * *\end{array}$ & $\begin{array}{l}0.5 \\
\pm 0.29\end{array}$ & $\begin{array}{ll}3.0 \quad \pm \\
0.41 *\end{array}$ & $\begin{array}{l}0.75 \pm \\
0.25 \\
\end{array}$ & $\begin{array}{l}3.75 \\
0.85^{*}\end{array}$ & $\begin{array}{ll}0.75 & \pm \\
0.25 & \end{array}$ & $\begin{array}{l}3.25 \\
0.48 \\
\end{array}$ \\
\hline
\end{tabular}

E.E $=$ end to end associations Total struct. Ex. gap = Total structural excluding gaps $*=\mathrm{P}<0.05 * *=\mathrm{P}<0.01$

Table (4): Mean values of different aberrations induced by acrylamide and the effect of antioxidants in rat spematocytes $(\mathrm{M} \pm \mathrm{SE})$

\begin{tabular}{|c|c|c|c|c|c|}
\hline \multirow{2}{*}{ Groups } & \multicolumn{5}{|c|}{ Types of aberrations } \\
\cline { 2 - 6 } & Chain & Auto & X-Y & Total & Ploidy \\
\hline control & $0.0 \pm 0.0$ & 0.25 & 0.75 & 1.25 & 0.25 \\
& & \pm 0.25 & \pm 0.25 & \pm 0.25 & \pm 0.25 \\
\hline ACR & 3.75 & 4.0 & 5.0 & 13.0 & 2.75 \\
& $\pm 0.63^{* * * * *}$ & $\pm 0.42^{* *}$ & $\pm 0.71^{* *}$ & $\pm 0.71^{* * *}$ & $\pm 0.48^{* *}$ \\
\hline barley & 0.25 & 0.75 & 1.0 & 2.75 & 0.75 \\
& \pm 0.25 & \pm 0.48 & \pm 0.41 & \pm 0.95 & \pm 0.48 \\
\hline ACR + barley & 1.25 & 1.5 & 2.5 & 5.25 & 1.25 \\
& $\pm 0.48^{*}$ & \pm 0.65 & \pm 0.87 & $\pm 1.03^{*}$ & \pm 0.69 \\
\hline Sage & 0.5 & 0.75 & 1.25 & 2.25 & 0.75 \\
& \pm 0.29 & \pm 0.25 & \pm 0.48 & \pm 0.48 & \pm 0.25 \\
\hline ACR + Sage & 2.25 & 3.0 & 3.5 & 9.0 & 1.75 \\
& $\pm 0.48^{*}$ & $\pm 0.41^{*}$ & $\pm 0.96^{*}$ & $\pm 0.91^{* *}$ & \pm 0.25 \\
\hline Sage + barley & 0.25 & 0.75 & 0.75 & 1.5 & 1.0 \\
& \pm 0.25 & \pm 0.25 & \pm 0.25 & \pm 0.65 & \pm 0.41 \\
\hline ACR + sage + barley & 0.75 & 1.0 & 2.25 & 4.25 & 1.75 \\
& \pm 0.48 & \pm 0.71 & \pm 1.0 & \pm 0.95 & \pm 0.63 \\
\hline
\end{tabular}

Auto $=$ autosomal univalents $\mathrm{X}-\mathrm{Y}=\mathrm{x}-\mathrm{y}$ univalents

$*=\mathrm{P}<0.05 * *=\mathrm{P}<0.01$ 


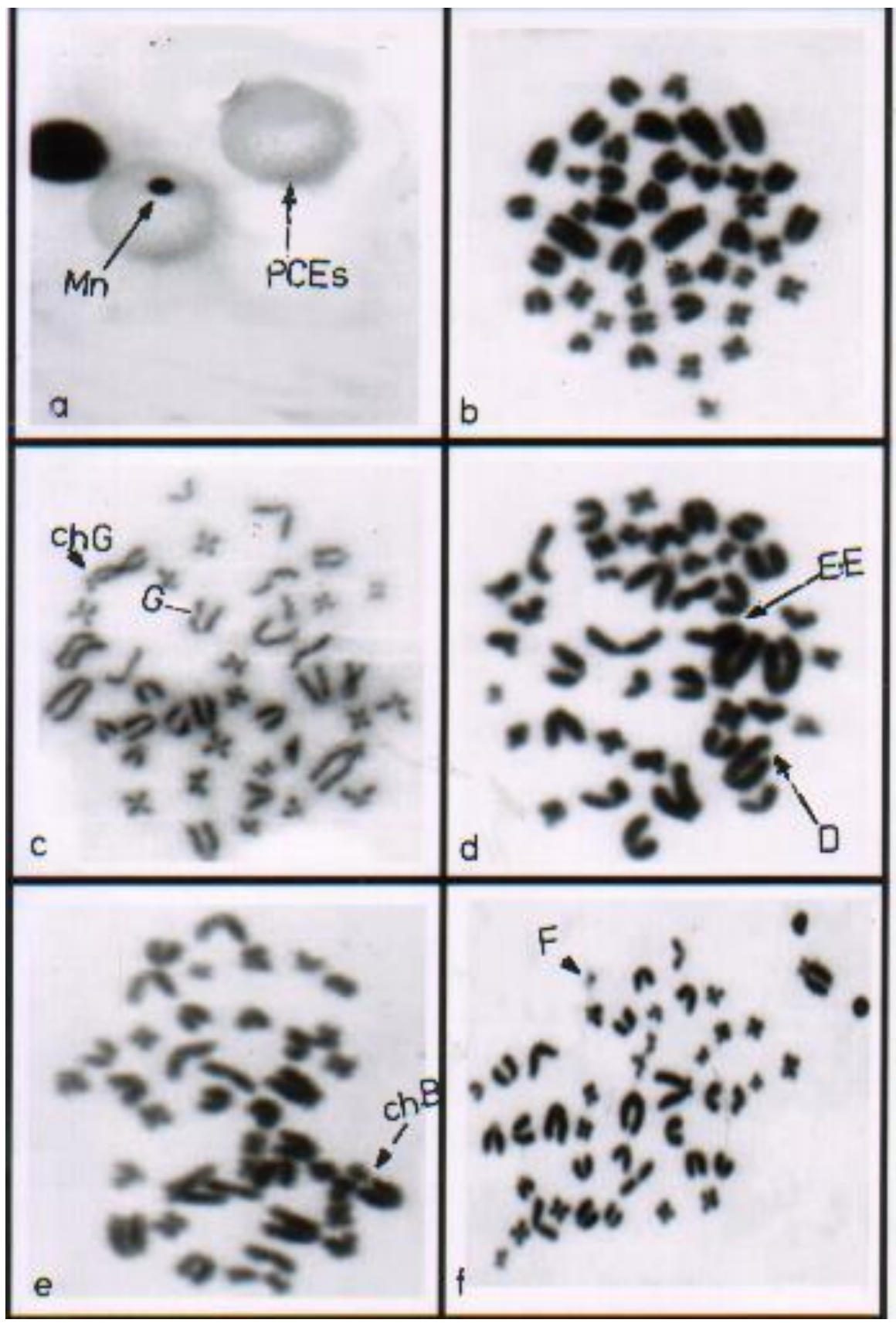

Fig. (1):

a- Photomicrograph of a rat bone marrow showing normal PCEs and contains micronucleus MnPCEs.

b- Normal rat metaphase spread

c- Metaphase spread of a treated rat with a gap (G) and chromosomal gap (ch.G)

d- Metaphase spread of a treated rat with a deletion (D) and end to end associations (E.E)

e- Metaphase spread of a treated rat with a chromosomal break (ch.B)

f- Metaphase spread of a treated rat with a fragment (F) 


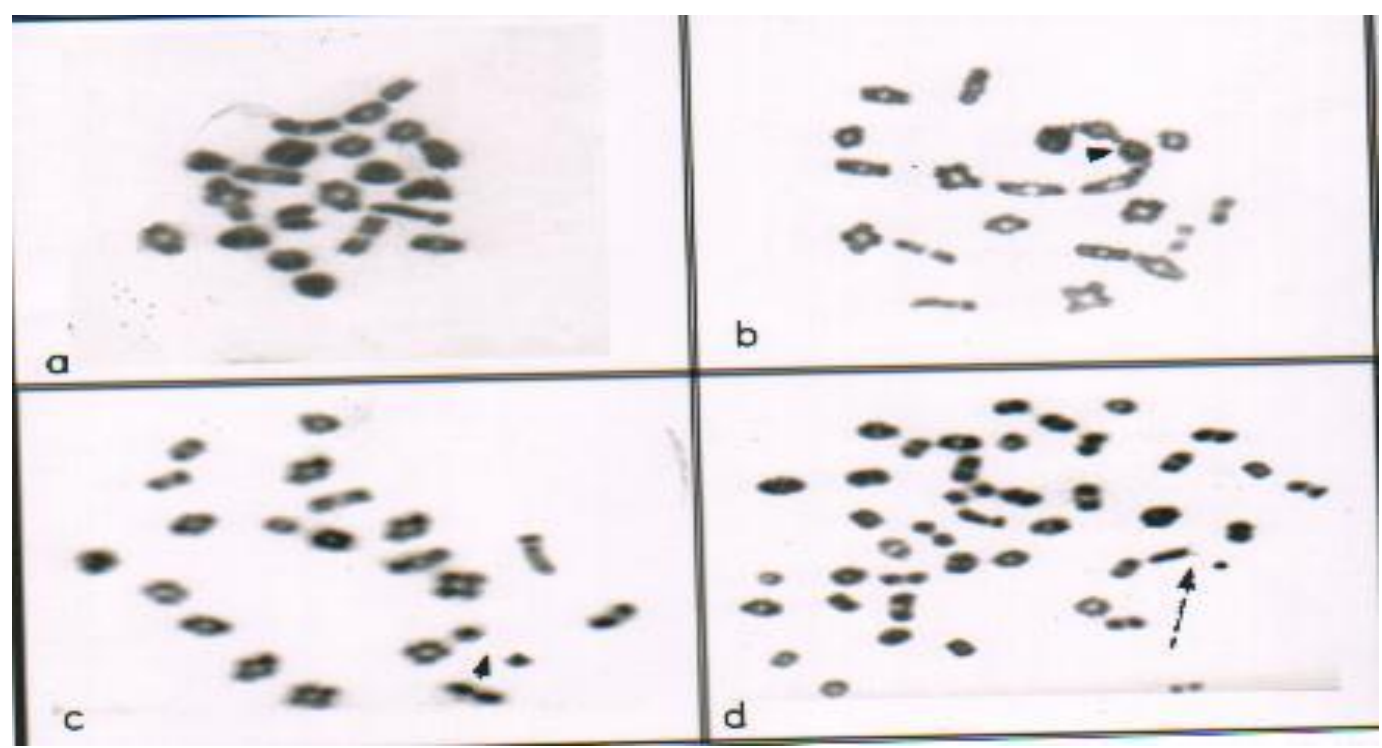

Fig (2):

a- A normal rat spermatocyte

b- Treated rat spermatocyte showing a chain

c- Treated rat metaphase spread showing autosomal univalents

d- Treated rat metaphase spread showing X-Y univalents and polyploidy

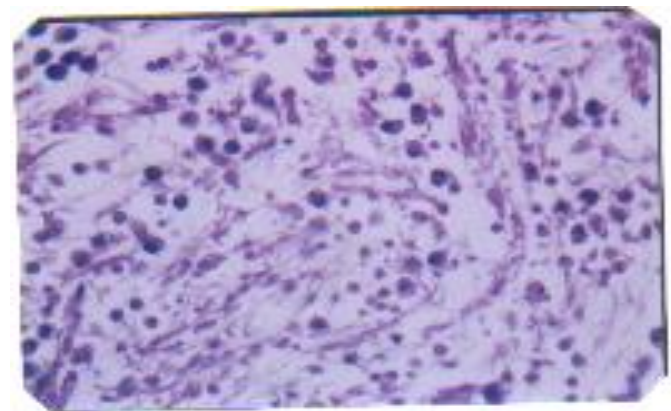

$\mathbf{a}$

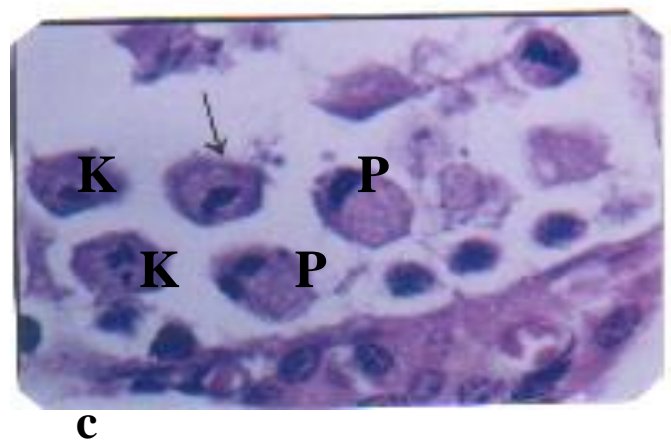

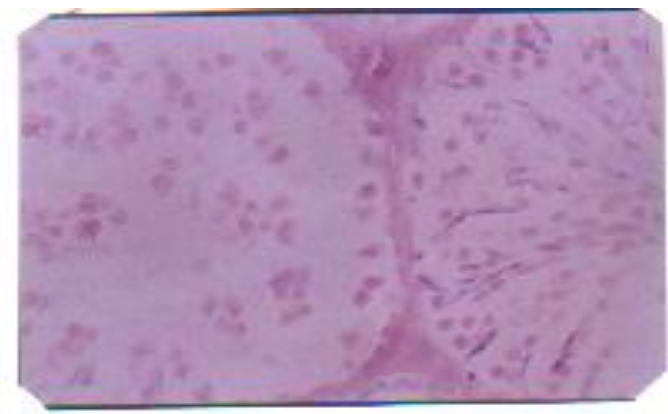

b

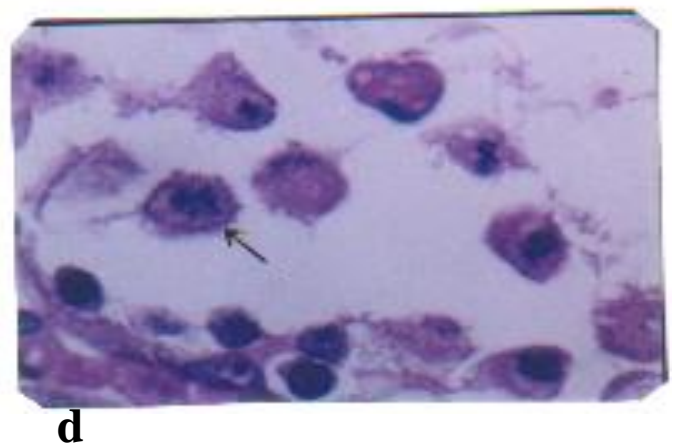

Fig. (3): Testis of albino rat orally exposed to acrylamide showing, (a) large number of residual bodies in the lumen of seminiferous tubules (H\&E x 400). (b) Remnants of mature spermatozoa in the lumen (Crystal violet $x$ 1000). (c) Different stages of necrosis in spermatocytes with pyknotic (p) and karyolytic (k) nuclei together with disturbed cytoplasm and nuclei of leydig cells (H\&E x 1000). (d) Prominent chromatolysis in the nuclei of spermatocytes (H\&E $x$ 1000) 


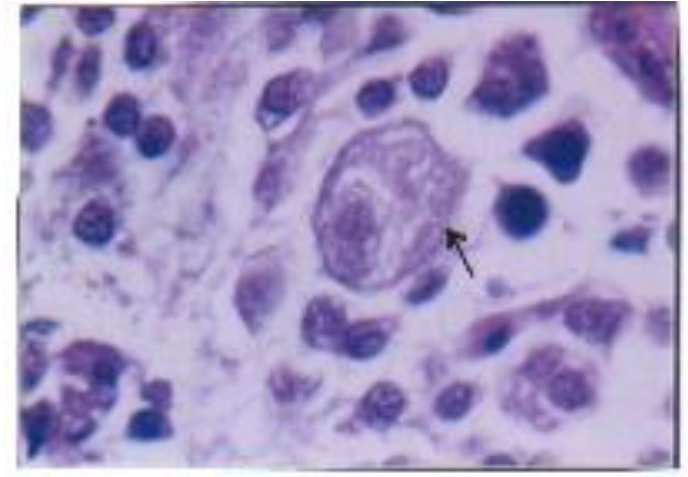

$\mathbf{a}$

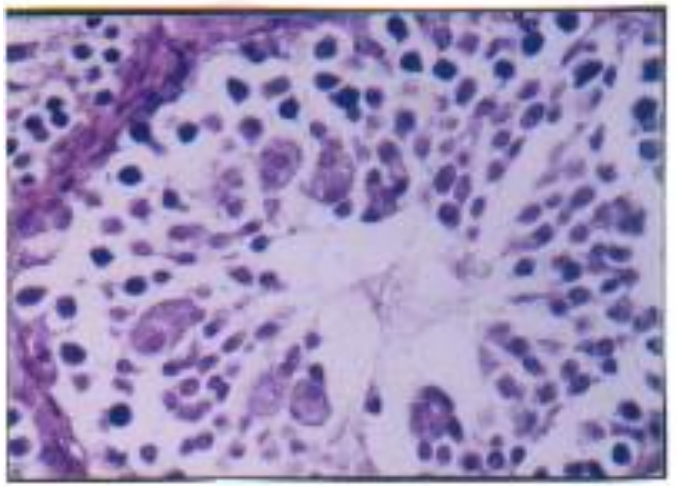

c

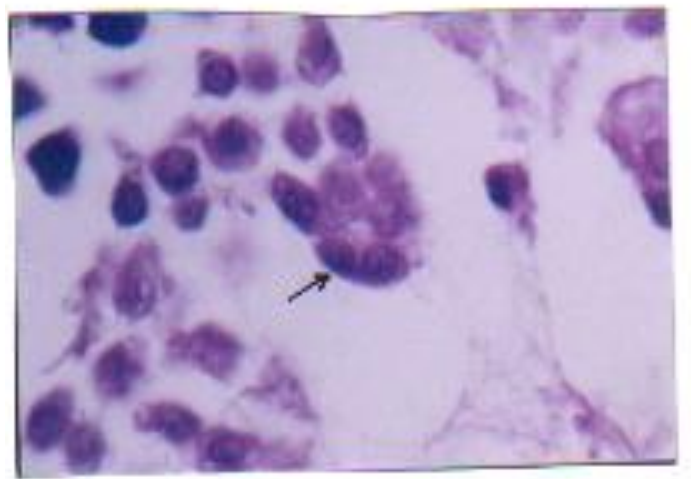

e

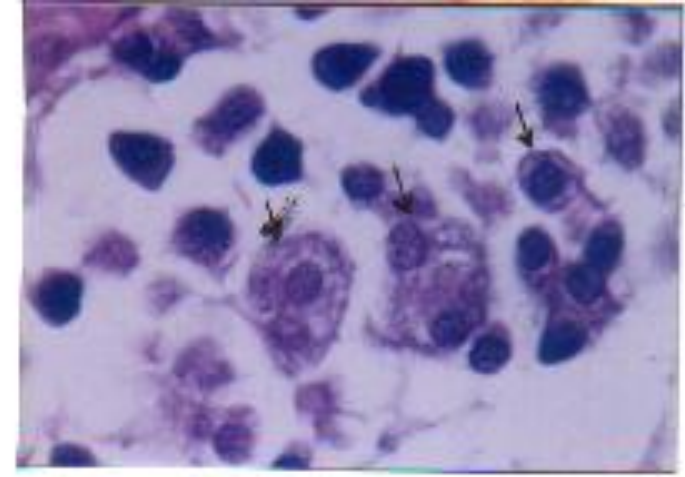

d

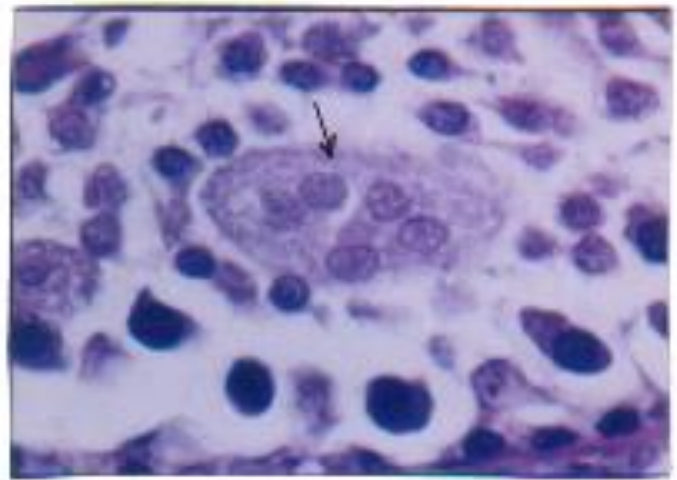

d

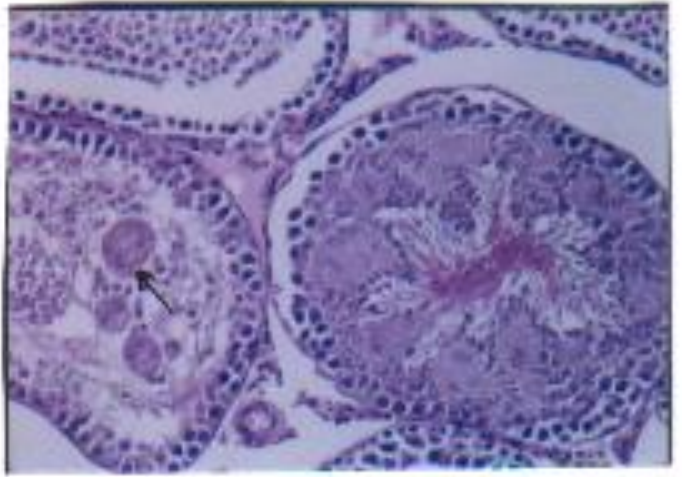

f

Fig. (4): Testis of rat treated orally with ACR showing (a) Spermatid giant cell with foamy cytoplasm and karyolytic nuclei (H\&E x 1000). (b) Few number of giant cells with two or three nuclei $(H \& E \times 1000)$. (c) Multiple spermatid giant cells in the lumen of seminiferous tubules (H\&E x 1000). (d) Multinucleated spermatid giant cell with five nuclei $(H \& E \times 1000)$. (e) Spermatid giant cell with three nuclei (H\&E x 1000). (f) Seminiferous tubules containing spermatid giant cells with marked interstitial oedema in between the shrunken semeniferous tubules

(H\&E x 200). 


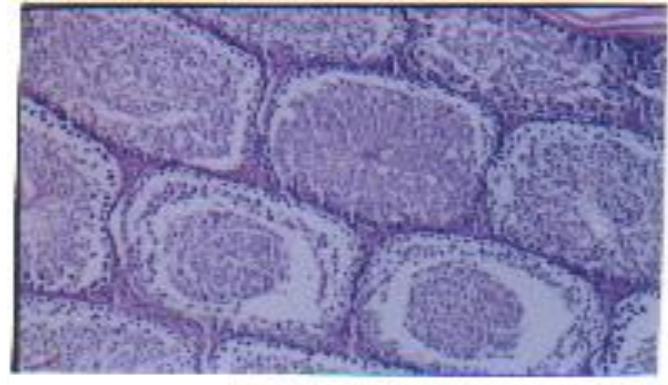

a

c

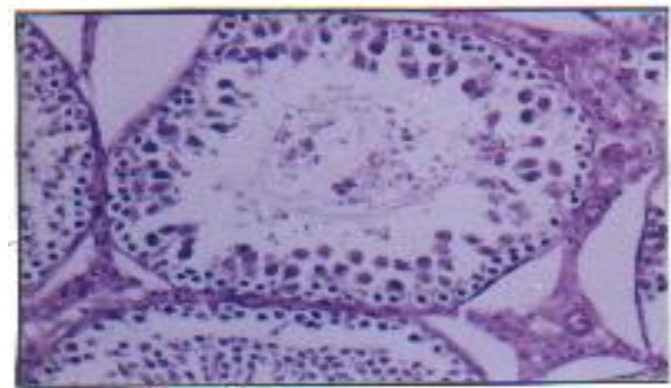

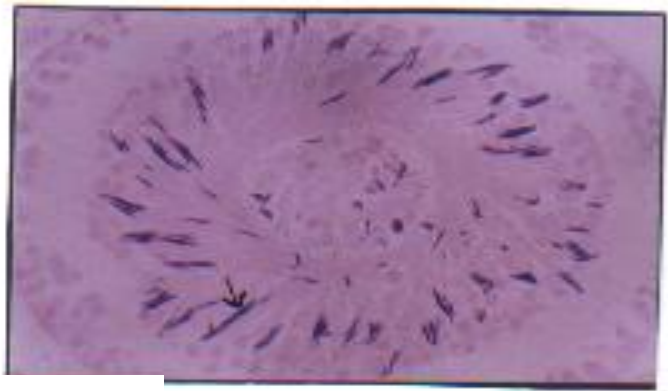

b

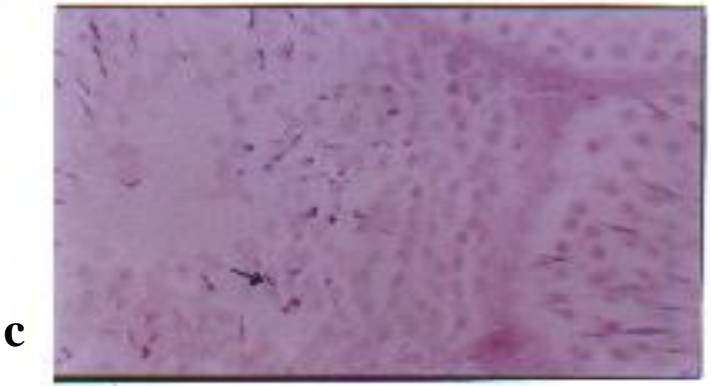

Fig. (5): Testis of rat fed barley or sage-supplemented diet and treated orally with ACR (a) ACR plus barley group showing some semeniferous tubules append normal, others appeared affected and contained disturbed spermatogenic activity (H\&E $x$ 100). (b) ACR plus barley showing large number of normal spermatozoa (Crystal violet stain $x$ 1000). (c) ACR plus sage showing few number of spermatozoa in the lumen and abnormal leydig cells (H\&E x 400). (d) ACR plus sage showing few number of normal spermatozoa in the lumen (Crystal violet stain $x$ 1000)
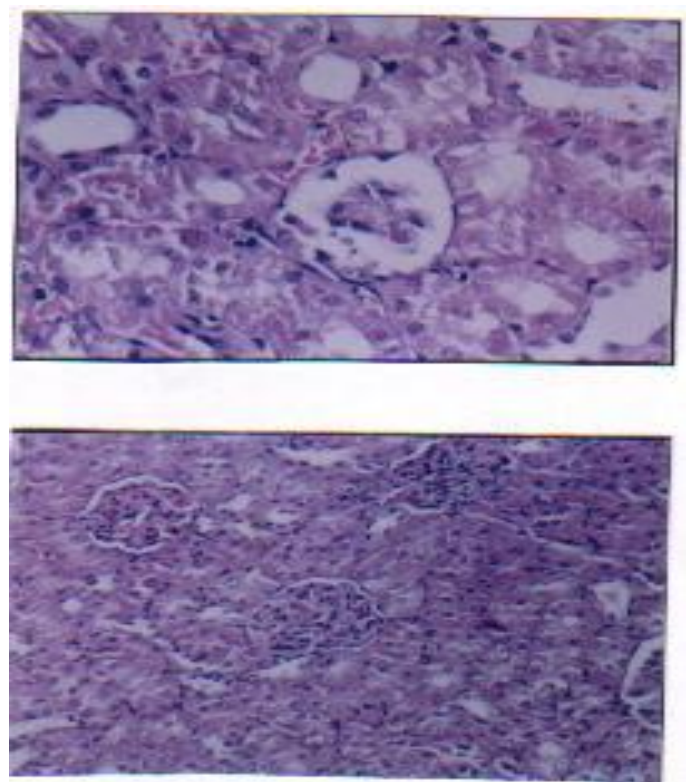

Fig. (6): (a) Kidney of rat treated orally with ACR showing mild degenerative changes with shrunken glomerular capillary tufts and distencted Bowman's capsules. Some proximal and distal convoluted tubules appeared highly affected . Debris of their nuclei could be detected (karyolysis) (H\&E x 400). (b) Treated with ACR plus barley and /or sage showing apparently normal tissue (H\&E x 200).. 


\section{Discussion}

ACR has recently been found in fried and baked foods, suggesting widespread public exposure (Erdreich and Friedman, 2004). The toxicity of ACR on human and experimental animals were well documented in a series of reports since the Swedish Food Administration alarm in 2002 (Konings et al., 2003, Lofstedt, 2003, LoPachin, 2004, Mucci et al., 2004, Ruden, 2004, Van Landingham et al., 2004). ACR has been shown to be carcinogenic in animals, and has been classified by the WHO/IARC among others as "probably carcinogenic for humans" this promoted us to investigate the protective effects of barley and sage against the ACR-induced toxicity and genotoxicity in rats. The selective dose of ACR was literaturaly based (Lehning et al., 2002). The LD50 values in rats were in the range 107-251 $\mathrm{mg} / \mathrm{kg}$ b.w (Shelby, 2004). In this study, the animals treated orally with ACR $(50 \mathrm{mg} / \mathrm{kg}$ b.w) for five days. The cumulative dose was comparable to the LD50 value reported in the literature. Our results indicated that ACR is a potent toxic material to the liver as indicated by the elevated levels of the biochemical parameters tested. Although, the only statistically and toxicologically significant decrease in serum-cholinestrase activity and increased serum alkaline phosphatase activity, there is some evidences that ACR induces an increase in the relative weight of the liver indicating that the liver tissues may be a target for the toxicity of ACR (Shelby, 2004).

The current study indicated that ACR induced micronuclei in polychromatic erythrocytes in bone marrow cells of rats. Similar to these results. Adler et al. (1988), Chihak and Vontorkova (1990) and Dobrzynska and Gajewski (2000) reported that ACR increased the micronucleus frequency in bone marrow cells in mice treated with $25 \mathrm{mg} / \mathrm{kg}$ b.w (two doses) or $50 \mathrm{mg} / \mathrm{kg}$ b.w (single dose). Significant increase in micronucleus frequency was also reported in reticulocytes and splenic lymphocytes from mice i.p. injected with ACR at doses of $50 \mathrm{mg} / \mathrm{kg} \mathrm{b.w}$ or more
(Backer et al. 1989; Kligerman et al. 1991; Russo et al. 1994).

The present study revealed that ACR increases significantly all types of chromosomal aberrations in somatic cells of rats. In the study of Marchetti et al. (1996), using cytogenetic analysis of first cleavage zygotes, almost all zygotes had at least one structural aberration after paternal treatment with ACR. Moreover, Cihak and Vontorkova (1988) found that a single i.p. injection of $100 \mathrm{mg} / \mathrm{kg} \mathrm{b} . \mathrm{w}$ ACR monomer elevated the frequency of chromosomal aberrations and micronucleated polychromatic erythrocytes in the bone marrow of male mice.

Our data revealed that ACR induced chromosomal aberrations in germ cells. In this regard, some literature data suggested that ACR mainly interact with protamines (Sega et al., 1989) whereas, other data suggested a direct interaction between ACR and DNA (Segerback et al., 1995). ACR is regarded as a potential mutagen based on experimental evidence that it can bind to DNA. Dearfied et al. (1988) suggested that ACR did not produce gene mutation. While ACR does not produce gene mutation in bacteria, but the epoxide metabolite glycidamide does in the absence of metabolic activation. This corroborates fairly with our findings which proved the role of ACR in producing the greatest impact of paternal ACR on abnormalities. We also suggest that chromatin adducts are likely to be converted into cytogenetic abnormalities when they are exposed to DNA repair.

According to Dearfield et al. (1988), ACR produced several genetic effects in germ cells such as chromosomal aberrations, micronuclei, sister chromatid exchange (SCE), polyploidy, aneuploidy and other mitotic disturbances. They also concluded that ACR impairs fertility in male rats, most likely through a direct toxic effect. In another study on germ cells, serial recovery of sperm from the caudate epididymides that were at the early spermatocyte (pre-meiotic/meiotic) stage to 


\section{Sekena, H. Abd El-Aziem et al}

the spermatozoa (post-meiotic) stage at the time of treatment showed no increase in unscheduled DNA synthesis (UDS); these stages correspond to those associated with dominant lethality (Sega et al. 1990). In contrast, DNA strand breaks were increased in these sensitive stages. DNA strand breakage in sperm in earlier stages of development at the time of treatment decreased with time, likely due to DNA repair, prior to become functional spermatozoa. Slight increase in DNA strand breaks in isolated human testicular cells have been reported. (Bjorge et al. 1996). Adler et al. (1988) and Backer et al. (1989), reported that chromosomal aberrations and sister chromatid exchanges were not found in spermatogonia or early spermatocytes in contrast to positive results in late spermatids and spermatozoa. Further studies of DNA, total sperm head, and sperm protamine alkylations (Sega et al. 1989) suggested that the stage specificity can be explained by preferential binding to cysteine sulfhydryl groups in sperm protamine. In mid-to late spermatid stages, chromosomal histones are replaced by protamines that are relatively rich in arginine and cysteine. Alkylation of free sulfhydryl groups of cysteine in the "immature" protamine of late spermatids and early spermatozoa might prevent normal chromatin condensation leading to stress in the chromatin structure and strand breakage.

The clastogenicity results observed in the present study were confirmed by the histological examination of the testis.

The antioxidant and protective effects of barley and sage have been documented previously (Kanauchi et al., 1998; Bandoniene et al., 2001; Dongowski et al., 2002; Perry et al., 2003). In the present study, addition of either barley or sage to ACR resulted in a significant improvement in the serum clinical chemistry. This may be due to the higher content of antioxidant substances in these plants. Several reports indicated that blood cholesterol and lipoprotein concentrations can be reduced in human (Braaten et al., 1994) and animal (Kalra and Jood, 2001) by â_glucan from barley. We also observed that feeding on barley diet reduced aberrations produced by ACR. Similar results were noticed by Deguchi et al. (2000) who revealed that the novel purple pigment hordeumin, an anthocyanin-tannin pigment in barley, decreased a reverse mutation from Trp-P-1, Trp-P-2, IQ, and B[_P. Moreover, hordeumin also decreased the reverse mutation from dimethyl sulfoxide extracts of grilled beef in Salmonella typhimurium TA98.

The present study indicated that sagesupplemented diet decreased ACR mutagenicity. Similar results were observed by Karakaya and Kavas (1999) who noticed a decrease in sodium azide mutagenicity in $S$ typhimurium TA 100 by sage. According to Farhat et al. (2001) the antioxidant activity of sage may be due to 1,8-cineole, the main constituent, although, other components included ketones such as camphor and alpha, beta-thujone, terpenes such as limonene and alpha, beta-pinenne, and alchhols such as borneol and linalool.

From pathological point of view, this study revealed testicular lesions in rats treated with ACR alone. These lesions were characterized by the presence of residual bodies in the semineferous tubules, decreased number of mature sperms and the presence of spermatid giant cells together with necrosis of spermatocytes. The necrotic spermatocytes appeared with pyknotic and karyolitic nuclei. Debris of most spermatogenic cells revealed disturbed architecture of the semineferous tubules which appeared devoid of sertoli cells.

The histological abnormalities in the testis reported by U.S EPA (2002) in animals treated with $150 \mathrm{mg} / \mathrm{kg}$ ACR appeared in spermatids particularly round spermatids (Golgi and cap phase) one day after treatment. Nuclear vacuolization and swelling were the most common lesions in the spermatids. Degeneration of spermatocytes and spermatogonia was also noted. By the second day after treatment, spermatid degeneration was most prominent. On day 3, multi-nucleated gaint cells were frequent. By day 7-10, clearing of the histologic abnormalities was evident. These pervious results supported our findings regarding the degenerative lesions in the testicular tissues and clearly indicated that 
ACR had toxic effects on male reproductive organs and also has a direct effect on leydig cells. In this concern, Kinomoto et al. (2000) evaluated the toxic effect of some chemicals on male reproductive organs and considered that, necrosis of spermatocytes and presence of spermatid giant cells as lesions indicating toxicity to male reproductive organs. On the other hand, decreased number of mature sperms in the lumen of semeniferous tubules that observed in our study indicated that ACR induces impairment of spermatogenesis either by direct action on spermatogonia or indirectly by affecting the integrity of the sertoli cells. However, the presence of spermatid giant cells indicated faulty in spermatid cell divisions. The faulty in the spermatid cell divisions and decreased fertility by ACR were mentioned by Chapin et al. (1995). In addition, Sakamoto and Hashimoto (1988) found that the main effects of ACR on testicular tissue were decreased sperm count and decreased number of spermatocytes.

The directed action of ACR on the testicular tissue could be explained according to the findings of Dearfield et al. (1988) who reported that ACR rapidly metabolized, primarily by glutathione conjugation and its toxic metabolites mainly persist in testis and skin.

In our study, the presence of residual bodies indicated degeneration of germ cells and spermatids where the residual cytoplasm of such cells phagocytosed by sertoli cells and appeared as residual bodies. The presence of residual bodies was completely explained by Gary et al. (1990). These pathological alterations were decreased when animals were treated with either barley or sage.

Concerning the histopathological findings in the renal tissue, the lesions were not severe in ACR- treated animals or the combined treatment with protective agents. These findings could be explained by the rapid excretion of the ACR ( $24 \mathrm{hrs}$.) post administration where the residues were detected in testis and skin only as mentioned by Dearfield et al. (1988). Adversely, previous studies indicated that the kidney is one of the organs affected by the higher dose of ACR in animal studies (EU, 2002)

In conclusion, the present study revealed that ACR induced toxic, clastogenic, and histological alterations typically to those reported in the literature. Both barley and sage have a protective role against these deleterious effects possibly due to their higher contents of antioxidant substances which can modulate the metabolism of ACR resulting in the reduction of its toxicity and/or increase the GSH production by the target organs which involved in the detoxification of ACR. These plants may be useful when add to certain foods cooked in a higher temperature.

\section{References}

1. ACGIH (1991). Acrylamide. Documntation of the threshold limit values and biological exposure indices, 12.

2. Adler, I.D., Ingwersen, I., Kliesch, U. and El Tarras, A. (1988). Clastogenic effects of acrylamide in mouse bone marrow cells. Mutat Res 206, 379-385.

3. Anzenbacher, P. and Anzenbacherova, E. (2001). Cytochromes P450 and metabolism of xeobiotics. Cell Mol. Life Sci. 58, 737-747.

4. Backer, L.C., Dearfield,K.L., Erexson, G.L.; Campbell, J. A., WestbrookCollins, B. and Allen, J. W. (1989). The effects of acrylamide on mouse germ-line and somatic cell chromosomes. Environ Mol Mutagen. 13, 218 -226.

5. Bancroft, D., Stevens, A. and Turner, R. (1996). Theory and Practice of Histological Techniques, Fourth edition, Churchill Livingstone, Edinburgh, London, Melbourne.

6. Bandoniene, D. Gruzdiene, D. and Vnskutions, P. R. (2001). Antioxidant activity of sage extracts in rapeseed oil irradiated with UV-rays. Nahrung 45, 105108.

7. Baricevic, D., Filipic, M., Tomazin, E., Cinc, M. and Zupancic, A. (1996). Evaluation of autochthonous and/or introduced genotypes of medicinal and aromatic plants in Slovenia (Salvia officinalis L.). In: Franz, Ch., Schulz, H., Schumann, G., Sharma, J.R. and Tatlioglu, T., Editors, 1996. Proceedings, Federal Centre for Breeding Research on Cultivated Plants, Quedlinburg, pp. 15-20. 
8. Bjorge, C., Brunborg, G. and Wiger, R.. (1996). A comparative study of chemically induced DNA damage in isolated human and rat testicular cells. Reprod Toxicol 10(6), 509-519.

9. Braaten, J. T., Wood, P. J., Scott,F. W., Wolynetz, M. S., Lowe, M. K., BradleyWhite, P. and Collins, M. W. (1994). Oat B-glucan reduces blood cholesterol concentration in hypercholesterolemic subjects. Eur. J. Clin. Nutr. 48, 465-474.

10. Chapin, R.E., Fail, P.A., George, J.D., Grizzle, T.B., Heindel, J.J., Harry, G.J., Collins, B.J. and Teague, J. (1995). The reproductive and neural toxicities of acrylamide and three analogues inSwiss mice, evaluated using the continuous breeding protocol. Fundam. Appl. Toxicol. 1, 9-24.

11. Charles, C.A. and Richmond, W. (1974). Enzymatic determination of total serum cholesterol. Clin. Chem., 20 , 4, 470-475 .

12. Chihak, R. and Vontorkova, M. (1990): Activity of acrylamide in single-, double-, and triple-dose mouse bone marrow micronucleus assays. Mutation Research 234, 125-127.

13. Cihak, R. and Vontorkova, M. (1988). Cytogenetic effects of acrylamide in the bone marrow of mice. Mutat Res. 209(1-2), 91-94.

14. Cherevaty,V.S., Vashhenko, T.N. and Shishkov, G.Z. (1980). Comparative evaluation of the antibacterial action of different extracts from Salvia officinalis. Rastitel'nye Resursy 16, 137- 139.

15. Chipault, J., Mizumo, G. and Lundberg, W. (1956). The antioxidant properties of spices in foods. Food Technology 10, pp. $209^{-} 211$.

16. Collins, B. W.; Howard, D. R. and Allen, J. W. (1992): Kinetochore-staining of spermatid micronuclei: studies of mice treated with X-radiation or acrylamide. Mutatg Res.281:287-294.

17. Dearfield, K. L., Abernathy, C. O., Ottley, M.S., Brantner, J. H. and Hayes, P.F. (1988). Acrylamide: its metabolism, developmental and reproductive effects, genotoxicity, and carcinogenicity. Mutat Res.195 (1),45-77.

18. Deeb, S. (1976). Affinity of normal and abnormal spermatozoa in the testis and semen to crystal violet - eosin stain.Proc. $13^{\text {th }}$ Arab Vet. Congr., 13-18 Nov., Cairo.

19. Deguchi, T., Yoshimoto, M., Ohba, R. and Ueda, S. (2000). Antimutagenicity of the purple pigment, hordeumin, from uncooked barley bran-fermented broth. Biosci. Biotechnol. Biochem. 64(2), 414416

20. Dobrzynska, M. M. and Gajewski, A. K. (2000). Induction of micronuclei in bone marrow and sperm head abnormalities after combined exposure of mice to low doses of $\mathrm{X}$-rays and acrylamide. Teratog. Carcinog Mutagen. 20(3), 133-140.

21. Dobrynin, V. N.; Kolosov, M. N.; Chernov, B. K. and Derbentseva, N. A. (1976). Antimicrobial substances of Salvia officinalis. Khimiya Prirodnykh Soedineii 5, 686-687.

22. Dongowski, G., Huth, M., Gebhardt, E. and Flamme, W. (2002). Dietary fiber-rich barley products benficially affect the intestinal tract of rats. J. Nutr. 132, 37043714.

23. Erdreich, L. S. and Friedman, M. A. (2004). Epidemiologic evidence for assessing the carcinogenecity of acrylamide. Regulatory Toxicology and Pharmacology 39, 150-157.

24. E.U.(European Union). (2002). EuropeanUnion Risk Assessment Report: Aceylamide. Institute for Health and Consumer Protection, 2002.

25. FAO/WHO. Health implications of acrylamide in food. (http://www.who. int/fsf/Acrylamide/Acrylamide_index.htm). Geneva, Switzerland: 2002.

26. Filipic, M. and Baricevic, D. (1997). Antimutagenic activity of Salvia officinalis extracts against UV-induced mutations in E. coli strains. Mutation Research 379 Suppl. 1, p. 182

27. Filipic, M. and Baricevic, D. ( 1998) Inhibitory effect of Salvia officinalis extracts on SOS functions induced by UVirradiation. Abstract Book of the 28th Annual Meeting of the Eurropean Environmental Mutagen Society (EEMS), Salzburg, p. 169.

28. Farhat, G. N., Affara, N. I. and GaliMuhtasib, H. U. (2001). Seasonal changes in the composition of the essential oil extract of East Mediterranean sage (Salvia libanotica) and its toxicity in mic. Toxicon. 39(10), 1061-1065.

29. Gary, A. B., Scot, L. E., Michael, R. E., Challes, A. M. and Villiam, F. M. (1990). Pathology of the Fischer rat : Rference and Atlas (Book). Academic pren inc. san Diego. California 92101.

30. Haisman, P. and Muller, B. R. (1977). Glossary of clinical chemistry terms. Butterworth, London, pp.126. 
31. Johansen, H. N., Wood, P. J. and BachKnudsen, K. E. (1993). Molecular weight changes in the (1---3),(1---4) â-D-glucan of Oats increased by the digestive processes in the upper gastrointestinal tract. J. Agri. Food Chem. 41, 2347-2352.

32. Johnson, K. A., Gorzinski, S. J., Bodner, K. M., Campbell, R. A., Wolf, C. H., Friedman, M. A. and Mast, R. W. (1986). Chronic toxicity and oncoenicity study on acrylamide incorporated in the drinking water of Fischer 344 rats. Toxicol. Appl. Pharmacol. 85, 154-168.

33. Kalra, S. and Jood, S. (2001). Effect of dietary barley b-glucan on cholesterol and lipoprotein fractions in rats. J. Cereal Sci. 31, 141-145.

34. Kanauchi, O., Mitsuyama, K., Saiki, T., Agata, K., Nakamura, T. and Iwanaga, T. (1998). Preventive effects of germinated barley foodstuff on methotrexate-induced enteritis in rats. Int. J. Mol. Med. 1(6), 961966.

35. Karakaya , S. and Kavas, A. (1999). Antimutagenic activities of some foods. Science Food and Agric. 79 (2), 237-242

36. Kinomoto, T., Sawada, M., Ogawa, S., Iguchi, A., Matsui, A., Iino, Y., Shiraishi, Y., Nishi, N. and Mera, Y. (2000). Collaborative work to evaluate toxicity on male reproductive organs by repeated dose studies in rats 3). Effects of repeated dose ethinylestradiol for 2 and 4 weeks on the male reproductive organs.J Toxicol. Sci.,:43-49.

37. Kligerman, A.D.; Atwater, A. L. and Bryant, M. F. (1991). Cytogenetic studies of ethyl acrylate using C57BL/6 mice. Mutagenesis 6(2), 137-141.

38. Konings, E. J., Baars, A. J., van Klaveren, J. D., Spanjer, M. C., Rensen, P. M., Hiemstra, M., van Kooij, J. A.A. and Peters, P. W. (2003). Acrylamide exposure from foods of the Dutch population and an assessment of the consequent risks. Food Ctem. Toxicol. 41(11), 1569-1679.

39. Lehning, E. J., Balaban, C. D., Ross, J. F., Reid, M. A. and LoPachin, R. M. (2002). Acrylamide neuropathy. I. Spatiotemporal characteristics of nerve cell damage in rat cerebellum. Neuro Toxicol. 23, 397-414.

40. Lofstedt, R. E. (2003). Science communication and the Swedish acrylamide “alarm". J. Health Commun. 8(5), 407-432.
41. LoPachin, R. M. (2004). The changing view of acrylamide neurotoxicity. Neuro Toxicology 25, 617-630.

42. Marchetti, F., Lowe, X., Moore, II. D., Bishop, J. and Wyrobek, A. (1996). Paternally transmitted chromosome structural aberrations detected in mouse first-cleavage zygote metaphases by multicolor FISHpainting. Chromosome Research. 8, 604-613.

43. Miller, M. J., Carter, D. E. and Sipes, I. G. (1982). Pharmacokinetics of acrylamide in Fisher-334 rats. Toxicol. Appl. Pharmacol. 63, 36-44.

44. Mucci, L. A., Lindblad, P., Steineck, G. and Adami, H. O. (2004). Dietary acrylamide and risk of renal cell cancer. Int. J. Cancer 109(5), 774-776.

45. Newall, C., Anderson, L.A. and Phillipson, J.D.( 1996). Sage. In: Newall, C.A., Anderson, L.A. and Phillipson, J.D., Editors, 1996: Herbal Medicines _ A Guide for Health-care Professionals, The Pharmaceutical Press, London.

46. Pearson, D.A., Frankel, E.N. and Edwin, N. (1997). Inhibition of endothelial cellmediated oxidation of low-density lipoprotein by rosemary and plant phenolics. Journal of Agricultural and Food Chemistry 45, 578_582

47. Perry, N. S., Bollen, C., Perry, E. K. and Ballard, C. (2003). Salvia for dementia therapy: review of pharmacological activity and pilot tolerability clinical trial. Pharmacol. Biochem. Behav. 75(3), 651659.

48. Preston, R. J., Dean, B. J.; Galloway, S., Holden, H., McFee, A. F. and Shelby, M. (1987). Mammalian in vivo cytogenetic assay. Mutat. Res. 189, 157-165.

49. Robertson,J. A., Masjak-Newman, G., Ring, S. G. and Selbevendran, R. R. (1997). (s24---4) â-D-glucan from barley: Effects of cooking and digestion. J. Cereal Sci. 25, 275-283.

50. Ruden, C. (2004). Acrylamide and cancer risk-expert risk assessments and the public debate. Food Chem. Toxicol. 42, 335-349.

51. Russo, A. (2000). In vivo cytogenetics: Mammalian germ cells. Mutt. Res. 455, 167-189.

52. Russo, A., Gabbani, G. and Simoncini, B. (1994). Weak genotoxicity of acrylamide on the premiotic and somatic cells of the mouse. Mutat. Res. 309, 263-272.

53. Sakamoto J, and Hashimoto K. (1988): Effects of N,N'-methylene-bis-acrylamide (MBA) on mouse germ cells--sperm count 


\section{Sekena, H. Abd El-Aziem et al}

and morphology, and testicular pathology. Arch Toxicol ;62(1):54-59.

54. Sakamoto, J., Kurosaka, Y. and Hashimoto, K. (1988). Histological changes of acrylamide-induced testicular lesions in mice. Exp. Mol. Pathol. 48, 324334.

55. Salamone, M., Heddle, J., Stuart, E. and Kotz, M. (1980). Towards as improved micronucleus test: Studies on 3 model agents. Mitomycin C. cyclophosphamide and dimethylbenzanthracene. Muttat. Res. 74, 347-356.

56. SAS Institute (1982). SAS User's Guide: Statistics. 1982 Edition, SAS Institute Inc., Cary, NC.

57. Sega, G., Generoso, E. and Brimer, P. (1990). Acylamide exposure induces a delayed unscheduled DNA synthesis in germ cells of male mice that iscorrelated with the temporal pattern of adduct formation in testis DNA. Environ. Mol. Mutagen 16, 137-142.

58. Sega, G.A., Valdivia Alcota, R.P., Tancongco, C.P. and Brimer, P.A.(1989). Acrylamide binding to the DNA and protamine of spermiogenic stages in the mouse and its relationship to genetic damage. Mutat. Res. 216, 221-230.

59. Segerback, D., Calleman, C. J., Schroeder, J. L., Costa, L. G. and Faustman, E. M. (1995). Formation of N7-(2-carbamoyl-2-hydroxyethyl) guanine in DNA of the mouse and rat following i.p administration of $\left({ }^{14} \mathrm{C}\right)$ acrylamide. Carcinogenesis 16(5), 1161-1165.

60. Shelby, M. D. (2004). NTP-cerhr panel report on the reproductive and developmental toxicity of Acrylamide. Center for the evaluation of risks to Human reprodu- ction. National Toxicology program, US Department of Health and Human Services. NTP-CERHR-Acrylamide-04.

61. Snedecor, G.W. and Cochran, W.G. ( 1961). Statistical methods. Iowa, U.S.A. The Iowa State Univ. Press, 593.

62. The committee on Enzymes of the Scandinavian Society for clinical chemistry and clinical physiology. (1974). Recommended methods for the determination of four enzymes in blood. Scand. J. Clin. Lab. Invest. 33, 291-306.

63. U.S. EPA. (1994). Chemical summary of acrylamide. Officee of Pollution Prevention and Toxic Substances, Washington DC.

64. U.S. EPA. (2002). United States Environmental Protection Agency Consumer Factsheet on: Acrylamide. EPA Office of Toxic Substances, Washington DC.

65. Van Landingham, C. B., Lawrence, G. A. and Shipp, A. M. (2004). Estimates of lifetime-absorbed daily doses from the use of personal-care products containing polyacrylamide: A Monte Carlo Analysis. Risk Analysis 24(3), 603-619.

66. Wahlefeld, A. W. (1974). In Methods of Enzymatic Analysis,vol.5,Bergmeyer, $\mathrm{H}$. U., ed. Academic Press, New York, pp. 1831-1835.

67. Waller, R. A. and Duncan, D. B. (1969). A Bayes rule for the symmetric multiple comparison problems. J. Am. Stat. Assoc. 64, 1484-1503.

68. Zenick, H., Hope, E. and Smith, M. K.(1986). Reproductive toxicity associated with acrylamide treatment in male and female rats. J Toxicol Environ Health. 17, 457-472. 


\section{الحماية من التغيرات الوراثية و البيوكيمائية و الههتوباثولوجية

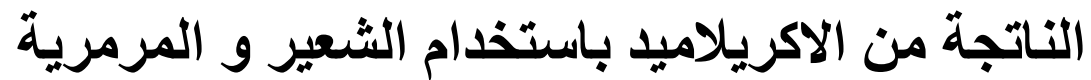

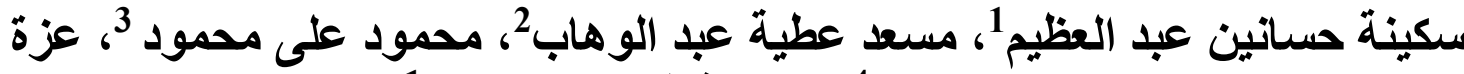

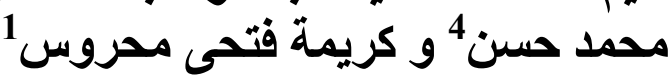

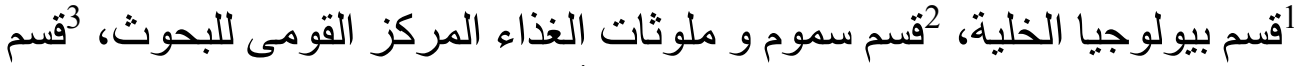

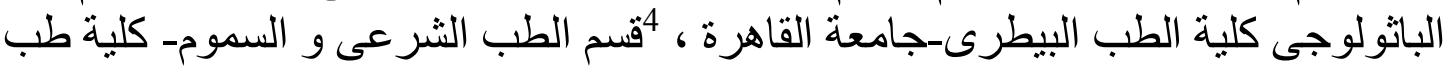

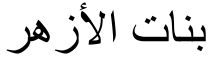

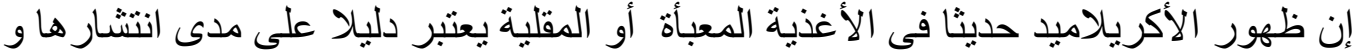

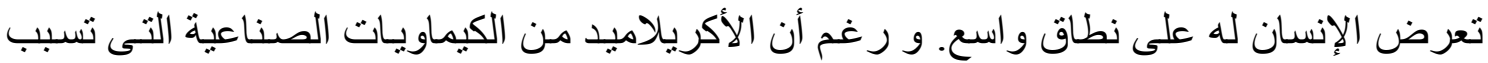

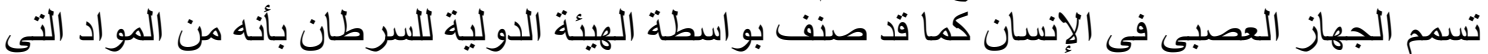

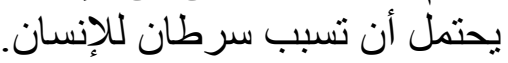

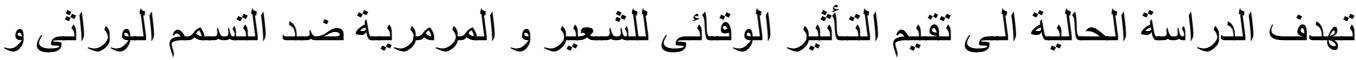

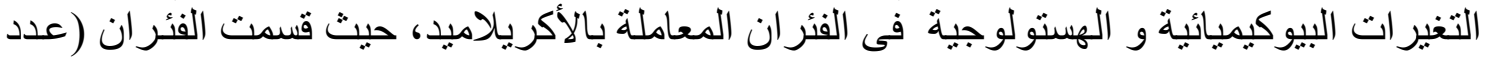

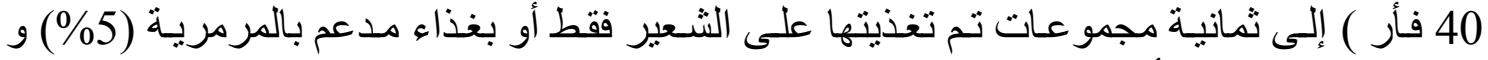

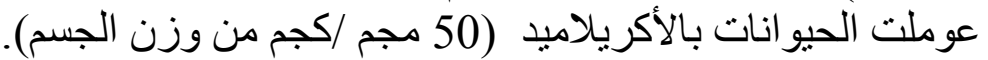

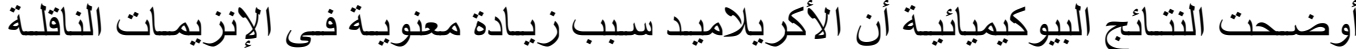

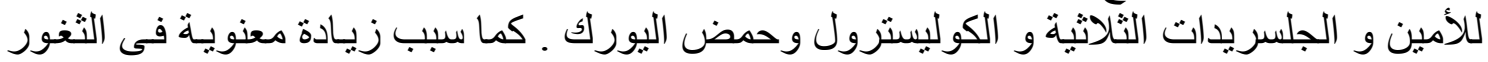

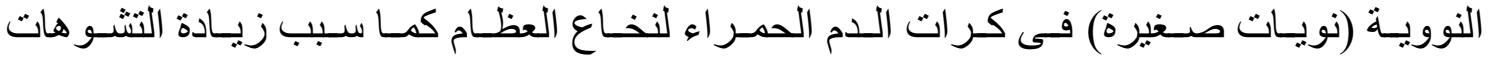

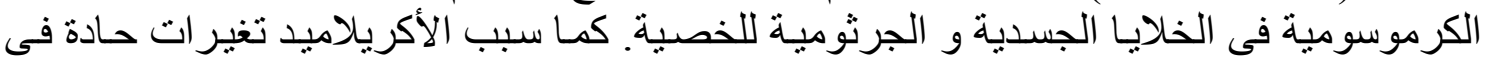

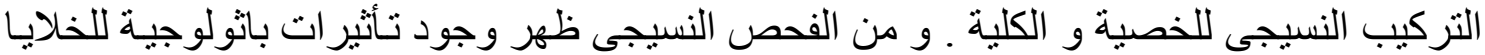

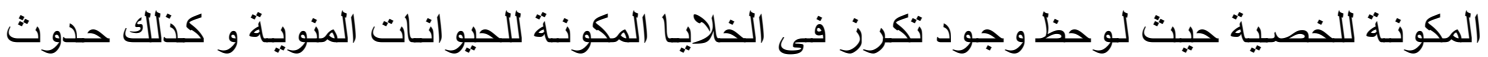

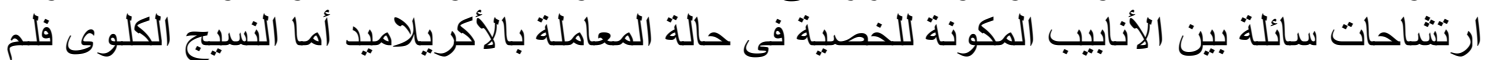
يحدث به تأثير ات خطيرة.

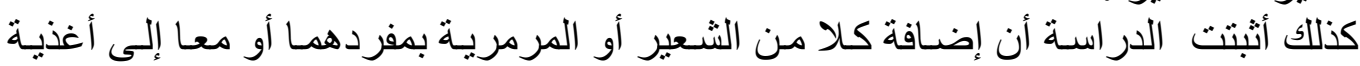

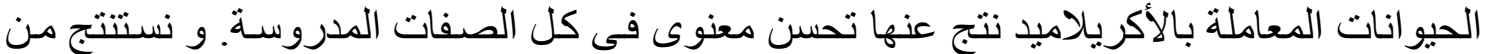

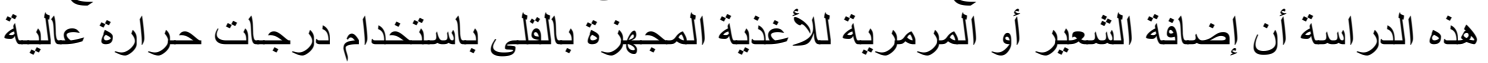
يمكن أن يكون له تأثير فعال فى حماية الإنسان من الأخطار الناتجة عن الأكريلاميد. 